\title{
Attention Problems Scale
}

National Cancer Institute

\section{Source}

National Cancer Institute. Attention Problems Scale. NCI Thesaurus. Code C121281.

A rating scale included in the Behavior Assessment System for Children that measures the tendency of the subject to be distracted and unable to concentrate. 